\title{
Mechanical and Pharmacological Revascularization Strategies for Prevention of Microvascular Dysfunction in ST-Segment Elevation Myocardial Infarction: Analysis from Index of Microcirculatory Resistance Registry Data
}

\author{
Ji-Hun Jang, ${ }^{1}$ Man-Jong Lee, ${ }^{1}$ Kyu-Yong Ko, ${ }^{1}$ Jin-Hee Park, ${ }^{1}$ Yong-Soo Baek, ${ }^{1}$ \\ Kwon Sung-Woo, ${ }^{1}$ Sung-Hee Shin, ${ }^{1}$ Seong-Ill Woo, ${ }^{1}$ Dae-Hyeok Kim, ${ }^{1}$ Young Ju Suh, ${ }^{2}$ \\ Jun Kwan, ${ }^{1}$ and Sang-Don Park $\mathbb{D}^{1}$ \\ ${ }^{1}$ Division of Cardiology, Department of Internal Medicine, Inha University Hospital, Incheon, Republic of Korea \\ ${ }^{2}$ Department of Biomedical Sciences, Inha University Hospital, Inha University School of Medicine, Incheon, Republic of Korea
}

Correspondence should be addressed to Sang-Don Park; denki1@inha.ac.kr

Received 22 August 2019; Revised 18 May 2020; Accepted 26 May 2020; Published 9 July 2020

Academic Editor: Andrea Rubboli

Copyright (c) 2020 Ji-Hun Jang et al. This is an open access article distributed under the Creative Commons Attribution License, which permits unrestricted use, distribution, and reproduction in any medium, provided the original work is properly cited.

Objectives. We aimed to identify mechanical and pharmacological revascularization strategies correlated with the index of microcirculatory resistance (IMR) in ST-elevation myocardial infarction (STEMI) patients. Background. Microvascular dysfunction (MVD) after STEMI is correlated with infarct size and poor long-term prognosis, and the IMR is a useful analytical method for the quantitative assessment of MVD. However, therapeutic strategies that can reliably reduce MVD remain uncertain. Methods. Patients with STEMI who underwent primary percutaneous coronary intervention (PCI) were enrolled. The IMR was measured with a pressure sensor/thermistor-tipped guidewire immediately after primary PCI. High IMR was defined as values $\geq 66^{\text {th }}$ percentile of IMR in enrolled patients (IMR $>30.9 \mathrm{IU}$ ). Results. A total of 160 STEMI patients were analyzed (high IMR $=54$ patients). Clinical factors for Killip class $(P=0.006)$, delayed hospitalization from symptom onset $(P=0.004)$, peak troponin-I level $(P=0.042)$, and multivessel disease $(P=0.003)$ were associated with high IMR. Achieving final thrombolysis in myocardial infarction myocardial perfusion grade 3 tended to be associated with low IMR $(P=0.119)$, whereas the presence of distal embolization was significantly associated with high IMR $(P=0.034)$. In terms of therapeutic strategies that involved adjusting clinical and angiographic factors associated with IMR, preloading of third-generation P2Y12 inhibitors correlated with reducing IMR value $(\beta=-10.30, P<0.001)$. Mechanical therapeutic strategies including stent diameter/length, preballoon dilatation, direct stenting, and thrombectomy were not associated with low IMR value (all $P>0.05$ ), and postballoon dilatation was associated with high IMR $(\beta=8.30, P=0.020)$. Conclusions. In our study, mechanical strategies were suboptimal in achieving myocardial salvage. Preloading of third-generation P2Y12 inhibitors revealed decreased IMR value, indicative of MVD prevention.

\section{Introduction}

ST-elevation myocardial infarction (STEMI) is usually caused by complete occlusion of a major epicardial coronary artery and results in myocardial ischemia and cell death. Early reopening of the culprit artery by primary percutaneous coronary intervention (PCI) is now considered as the cornerstone of the treatment [1-3]. However, although patients with STEMI restored normal coronary flow after primary PCI in many cases, they failed to achieve myocardial microvascular reperfusion, resulting in poor clinical outcome $[4,5]$. Many previous studies demonstrated that coronary microvascular dysfunction (MVD) was correlated with infarct size, and the presence of MVD was associated with an increased risk of cardiovascular events. Distal embolization of atheromatous debris, swelling of cardiomyocytes associated with interstitial edema, and reperfusion-related myocardial injury after primary PCI is 
considered as the major mechanism of microvascular damage [5-10].

The index of microcirculatory resistance (IMR) can provide a quantitative assessment of the microvascular function of epicardial stenosis and hemodynamic condition independently. IMR is considered an independent, powerful predictor of microvascular damage in STEMI [11-15]. Recent studies demonstrated that IMR assessed immediately after primary PCI is well correlated with the recovery of left ventricular function in STEMI $[1,7]$.

Through imaging tools or invasive coronary physiological indices, many studies have attempted to prove the therapeutic strategies of mechanical and pharmacological revascularization that can reduce microvascular damage from myocardial infarction. However, there are no definitive treatments for myocardial infarction that can reliably reduce microvascular damage, and some current therapeutic strategies remain controversial $[4,16-18]$.

Therefore, we aimed to identify mechanical and pharmacological revascularization strategies that prevent MVD in patients with STEMI using IMR that can assess microvascular integrity with primary PCI.

\section{Materials and Methods}

2.1. Study Population. We retrospectively reviewed consecutive patients with STEMI who underwent primary PCI with coronary physiological measurements between May 2009 and June 2016 at the clinic of a tertiary referral center (Inha University Hospital, South Korea).

STEMI was diagnosed in patients with symptoms that presented as myocardial ischemia with ST-segment elevation on electrocardiography (ECG) and subsequent release of cardiac biomarkers. ST-elevation was defined as new ST elevation at $J$ point in two contiguous leads of $\geq 2 \mathrm{~mm}$ $(0.2 \mathrm{mV})$ in men or $\geq 1.5 \mathrm{~mm}(0.15 \mathrm{mV})$ in women in leads $\mathrm{V} 2 \sim 3$ and/or of $\geq 1 \mathrm{~mm}(0.1 \mathrm{mV})$ in other contiguous chest leads of the limb leads $[2,3]$. We enrolled the patients who had developed STEMI within 12 hours (symptom to hospital time $<12$ hours) and had been successfully treated by primary PCI of the infarct-related artery with a modern drugeluting stent. The patients who had unprotected left main coronary artery disease or the culprit lesion at a side branch, stent thrombosis, high-degree atrioventricular block, cardiogenic shock with Killip class IV, contraindication to adenosine, previous cerebrovascular accident or myocardial infarction, and final thrombolysis in myocardial infarction (TIMI) grade $0 / 1$ were excluded.

This study design was approved by the Institutional Review Board of Inha University Hospital, Incheon, South Korea, and was conducted in compliance with the ethical principles outlined in the Declaration of Helsinki (INHAUH2020-04-057). The written informed consent was obtained from all patients.

\subsection{Echocardiographic Analysis and Cardiac Biomarkers.} Transthoracic echocardiography was performed using commercially available instruments less than 24 hours after primary PCI. Echocardiographic parameters were measured following the American Society of Echocardiography guidelines [19]. Left ventricular ejection fraction (LVEF) was calculated by the biplane Simpson method. Regional wall motion abnormality was obtained according to the recommendations of current guidelines. An experienced cardiologist who had no information of IMR accessed the wall motion score index (WMSI). WMSI was assessed in a 16segment model and calculated as the sum of all scores divided by the number of segments visualized. LV mass and LV mass indexed to body surface area were estimated by LV cavity dimension and wall thickness at end-diastole.

Baseline $N$-terminal pro- $B$-type natriuretic peptide (NTproBNP) level was checked, and cardiac enzymes were measured before and after PCI and the highest value during follow-up was assessed.

2.3. Angiographic Analysis and Treatment Strategies for STElevation Myocardial Infarction. All patients received preloading of dual-anticoagulation drugs (300 $\mathrm{mg}$ of aspirin and $600 \mathrm{mg}$ of clopidogrel or $180 \mathrm{mg}$ of ticagrelor or $60 \mathrm{mg}$ of prasugrel) before primary PCI. All patients were administered intravenous continuous infusion (100 IU/Kg) following a bolus injection of unfractionated heparin (5,000 IU). Intravenous morphine was used depending on the clinician's decision before or during PCI. After a loading dose of anticoagulation, primary PCI was performed in accordance with the current guideline [3]. A full range of commercially available guiding catheters, balloon catheters, and guide wires were used. Pre/postballoon dilation, direct stenting, or thrombus aspiration was performed based on the physician's discretion. Thrombus aspiration was performed from $>2$ passages across the lesion using an Export Advance aspiration catheter (Medtronic, Inc., Minneapolis, MN, USA) before stent insertion. Glycoprotein (GP) IIb/IIIa inhibitors were administered as bailout therapy in the events of slowor no-reflow phenomenon after revascularization. Pre- and post-TIMI grade and TIMI myocardial perfusion grade (TMPG) were assessed using grades $0-3$ based on final cine images obtained after a successful primary PCI. TIMI thrombus grade, collateral flow grade, and presence of distal embolization were obtained from the angiographic findings $[20,21]$. Distal embolization was defined as a distal filling defect with a sudden "cut-off" in one of the coronary branches of the culprit artery, distal to the angioplasty site [9].

Stent size/diameter and periprocedural techniques including pre/postballoon dilatation, direct stenting, and thrombus aspiration were defined as "mechanical strategies." "Pharmacologic strategies" included loading of dual antiplatelet, use of GP IIb/IIIa inhibitors, and morphine use.

2.4. Physiological Assessment: IMR Study. IMR was measured with a pressure sensor/thermistor-tipped guide wire (Radi Pressure Wire 5; Radi Medical Systems, Uppsala, Sweden) at the culprit lesion immediately after primary PCI (Figure 1). The pressure sensor/thermistor-tipped guide wire was initially calibrated outside the body and positioned in 


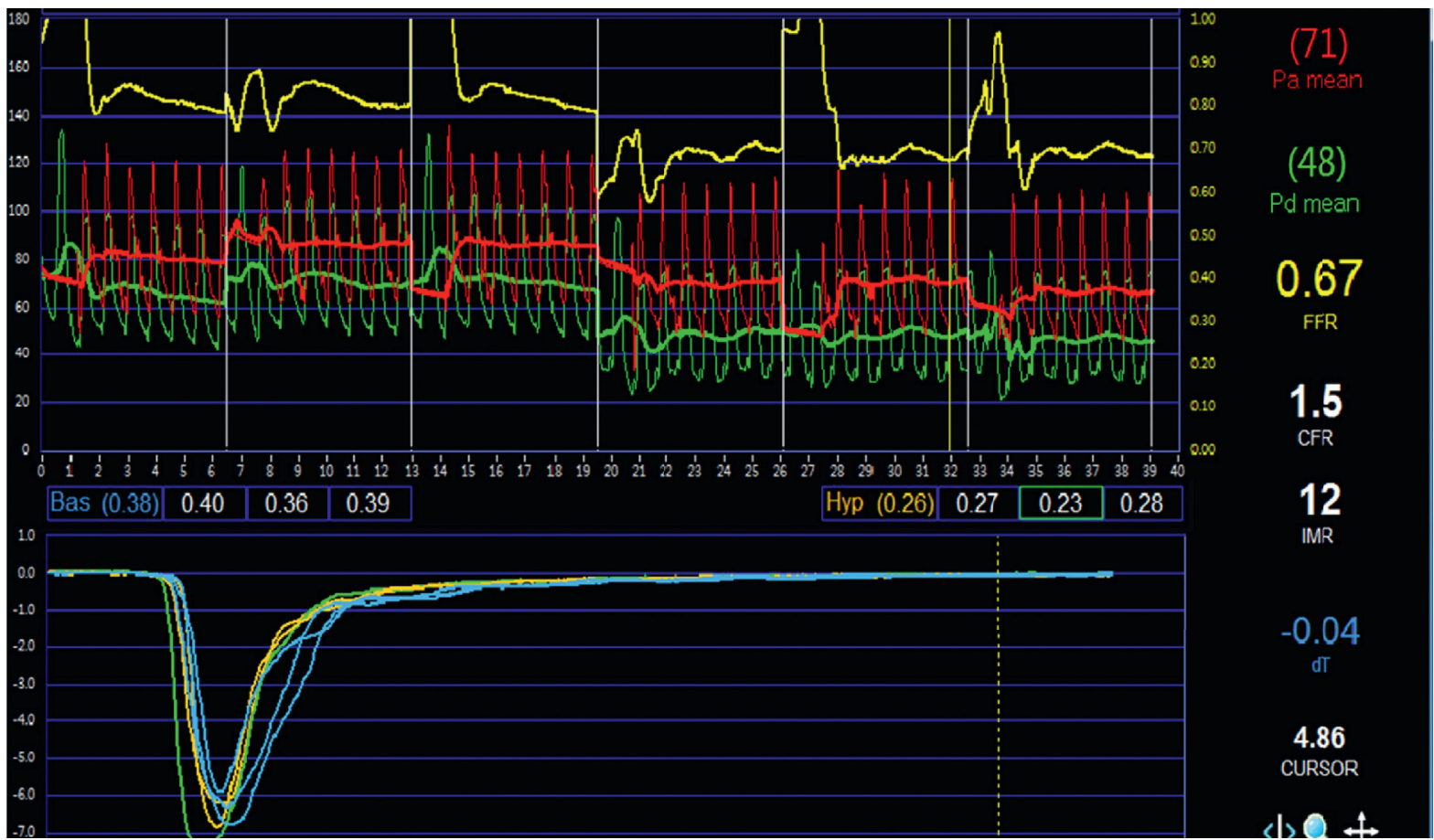

Figure 1: Thermodilution curves under resting conditions (yellow lines) and during hyperemia (blue lines) induced by intravenous adenosine infusion. A graphical representation of the baseline and hyperemia (seconds) thermodilution curves is seen in the catheterization lab at the time of percutaneous coronary intervention that was displayed on the RADI analyzer (Radi Medical Systems, Uppsala, Sweden).

the distal two-thirds of the culprit vessel after equalizing to the guiding catheter $[12,22]$. Intracoronary nitroglycerine $(200 \mu \mathrm{g})$ was administered and hyperemia was induced by administration of adenosine infusion $(140 \mu \mathrm{g} / \mathrm{kg} \bullet \mathrm{min})$ via femoral or antecubital vein. After achieving maximal hyperemia, the mean hyperemic transit time was recorded by averaging the value after rapid injection of $3 \mathrm{~mL}$ of roomtemperature saline through the coronary catheter. At the same time, mean aortic pressure $(\mathrm{Pa})$ and distal coronary arterial pressure $(\mathrm{Pd})$ were obtained. Fractional flow reserve (FFR) was calculated by using the following equation:

$$
\text { FFR }=\frac{\text { distal coronary pressure }}{\text { aortic pressure }} \text {. }
$$

The IMR was calculated as using the following equation: $\mathrm{IMR}=$ distal pressure $\times \mathrm{Tmn}$ (mean transit time) during hyperemia.

Additionally, thermodilution coronary flow reserve (CFR) was calculated using the following equation:

$$
\mathrm{CFR}=\frac{\text { hyperemic } \mathrm{Tmn}}{\text { resting } \mathrm{Tmn}} .
$$

Vessels with severe stenosis (distal coronary pressur$\mathrm{e} \leq 60 \mathrm{mmHg}$ ) and collateral flow were excluded from the analysis [22]. All IMR values were corrected by using Yong's formula (corrected IMR (IMRcorr) $=$ Pa $\times$ Tmn $\times([1.35 \times \mathrm{Pd} /$ $\mathrm{Pa}]-0.32)$. We defined "High IMR" as values $\geq 66$ th percentile of IMRcorr in the enrolled patients. In our study, "High IMR" was IMRcorr $\geq 30.9$ U.
2.5. Clinical Outcome. We assessed 3-year major advanced cardiac events (MACE) for the study population. MACE was defined as the composite of cardiovascular (CV) death, hospitalization because of heart failure (HF), target lesion revascularization (TLR), stent thrombosis, nonfatal myocardial infarction (MI), stroke, and major bleeding.

2.6. Statistical Analyses. Data were expressed for continuous variables as mean \pm standard deviation (SD) or median with interquartile range (IQR), as needed. Categorical variables were expressed as counts and percentages. Student's t-test and Pearson's Chi-square test were used to compare each parameter as needed. The Mann-Whitney $U$ test was used for skewed variables, and Fisher's exact test was used when the expected frequency was lower than 5. To determine the variables related to IMR value, linear regression analysis was performed. The final multiple regression model was made by stepwise forward regression based on a $P$ value of 0.2 and the clinical significance. The therapeutic strategies associated with IMR value were analyzed using multiple linear regression analysis. In addition, therapeutic strategies that were significantly associated with IMR value were adjusted for parameters that considered significant relation with IMR value on the previous multivariate model.

For all tests, a $P$ value less than 0.05 was considered statistically significant. All statistical analyses were performed with $R$ statistical software (version 3.4.1; $R$ Foundation for Statistical Computing, Vienna, Austria). 


\section{Results}

3.1. Baseline Characteristics. Of the 804 patients with STEMI, 210 patients could not perform coronary physiological measurements according to the exclusion criteria. Additionally, 421 patients did not agree with the IMR study or were unable to provide informed consent. Finally, 160 patients were enrolled in our study (Figure 2).

The baseline clinical data are as follows (Table 1). The overall mean age was $56 \pm 11$ years, 141 patients $(88.1 \%)$ were men, and 6 patients (3.8\%) showed Killip class III upon admission.

The High IMR group was older than the Low IMR group (60 \pm 11 years versus $54 \pm 11$ years, $P=0.001)$. Medical history was comparable between the two groups. While there was no difference for the door to balloon time, the High IMR group showed delayed hospitalization after symptom development (144.0 [71.0-360.0]) min versus 95.0 [(60.0-174.0] $\mathrm{min}, P=$ 0.003 for symptom onset to hospital time) and delayed revascularization $(235.0 \quad[159.0-397.0] \quad \mathrm{min}$ versus 162.0 [115.0-265.0] $\mathrm{min}, P=0.001$ for symptom onset to balloon time). The High IMR group showed a greater decrease in LV systolic function (LVEF, $44.7 \pm 6.8 \%$ versus $47.3 \pm 7.0 \%$, $P=0.028)$ and elevated E/e' (10.5 [9.3-12.5] versus 9.8 [8.0-11.2], $P=0.024)$. WMSI was more elevated in the High IMR group (1.7 [1.3-1.8] versus 1.5 [1.2-1.8], $P=0.040)$. The peak levels of creatine kinase (CK) and creatine kinase muscle/ brain (CK-MB) were more elevated in the High IMR group (peak CK, 3102.0 [1406.0-5580.0] IU/L versus 2020.0 [729.0-3897.0] IU/L, $P=0.028$; peak CK-MB, 289.4 [129.0-465.9] $\mathrm{ng} / \mathrm{mL}$ versus 188.0 [73.0-334.0] $\mathrm{ng} / \mathrm{mL}$, $P=0.010)$, whereas troponin-I level was comparable $(P=0.084)$. NT-proBNP level was higher in the High IMR group but statistically nonsignificant.

3.2. Coronary Angiography and Physiologic Studies. The results of coronary angiography and physiological studies are displayed in Table 2. The multivessel disease was more frequent in the High IMR group $(53.7 \%$ vs. $30.2 \%$, $P=0.006)$. However, there was no significant difference in the vessel territory of the culprit lesion between the two groups. A hundred patients (62.5\%) showed initial TIMI grade 0/1 flow before PCI, 144 patients (90.0\%) achieved final TIMI grade 3 flow, and 112 patients (70.0\%) showed final TMPG 3. Twenty-six (16.2\%) patients showed distal embolization and High IMR group was observed more often (31.5\% versus $8.5 \%, P<0.001)$. Fifteen patients $(9.4 \%)$ showed no-reflow phenomenon during PCI. The High IMR group could not achieve final TIMI 3 flow and final TMPG 3 compared with the Low IMR group (77.8\% versus $96.2 \%$, $P=0.001$ and $46.3 \%$ versus $82.1 \%, P<0.001$, respectively). Although TIMI thrombus grade and collateral flow grade were comparable between the two groups, the presence of distal embolization was greater in the High IMR group (14.8\% versus $6.6 \%, P<0.001)$.

In physiological assessment, there were significant differences in resting Tmn and hyperemic Tmn (0.7 [0.6-1.0] versus $0.4[0.3-0.6], P<0.001 ; 0.6[0.4-0.8]$ versus 0.2
[0.2-0.3], $P<0.001$, respectively). The overall value of FFR was similar between the two groups, whereas CFR was lower in the High IMR group (1.3 [1.0-1.8] versus 1.8 [1.2-2.6], $P<0.001)$. The overall median IMR was $22.4[13.9-35.0] \mathrm{U}$.

3.3. Clinical Outcomes of the Study Population. As 7 patients were lost during follow-up, we analyzed 153 patients for 3year MACE. Three-year MACE occurred in 17 (11.1\%) patients and were comparable between the two groups (15.4\% versus $8.9 \%, P=0.279)$. CV death occurred in 3 (2.0\%) patients who were included only in the High IMR group $(P=0.038)$. Other events including hospitalization for HF, TLR, stent thrombosis, nonfatal MI, stroke, and major bleeding were comparable (Table 3 ).

3.4. Therapeutic Strategies for STEMI. Mechanical and pharmacological treatment strategies were accessed in the study population (Table 4). All patients were implanted with third-generation drug-eluting stents. The stent size and length were comparable between the two groups. There was no significant difference in the treatment of balloon angioplasty $(68.5 \%$ versus $76.4 \%, P=0.377$ for preballoon dilatation; $16.7 \%$ versus $10.4 \%, P=0.376$ for postballoon dilatation). Forty-two patients (26.2\%) underwent direct stenting and 75 patients (46.9\%) underwent aspiration thrombectomy. Thirty-one patients (19.4\%) underwent a combination of aspiration thrombectomy with GP IIb/IIIa inhibitors. All mechanical therapeutic strategies showed no significant difference between the two groups.

All patients received preloading with $300 \mathrm{mg}$ of aspirin. The High IMR group showed a higher incidence of clopidogrel use than third-generation P2Y12 inhibitors $(79.6 \%$ versus $60.4 \%, P=0.023)$. The GP IIb/IIIa inhibitor was more frequently used in the High IMR group than in the Low IMR group $(27.8 \%$ versus $17.9 \%, P=0.216)$. In terms of morphine use, both groups showed almost equal proportions (51.9\% versus $64.2 \%, P=0.183$ ).

3.5. Predictors of Microvascular Dysfunction and Therapeutic Strategies Preventing Microvascular Dysfunction. As a result of analyzing the clinical, echocardiographic, and angiographic parameters, higher Killip class, delayed symptomonset-to-hospital time, higher peak troponin-I level, presence of multivessel disease, and occurrence of distal embolization were correlated with increasing IMR value (Table 5).

We analyzed each therapeutic strategy for the association of IMR value using linear regression analysis (Table 6). In mechanical strategies, increasing stent diameter was not significantly associated with low IMR value $(\beta=-8.00$, $P=0.057)$. Longer stent length and postballoon dilatation were associated with higher IMR value $(\beta=0.31, P=0.046$ and $\beta=10.44, P=0.015$, respectively). Preballoon dilatation, direct stenting, and thrombectomy showed no significant association with IMR value. In pharmacological strategies, the loading of third-generation P2Y12 inhibitors was significantly associated with low IMR value $(\beta=-7.24$, 


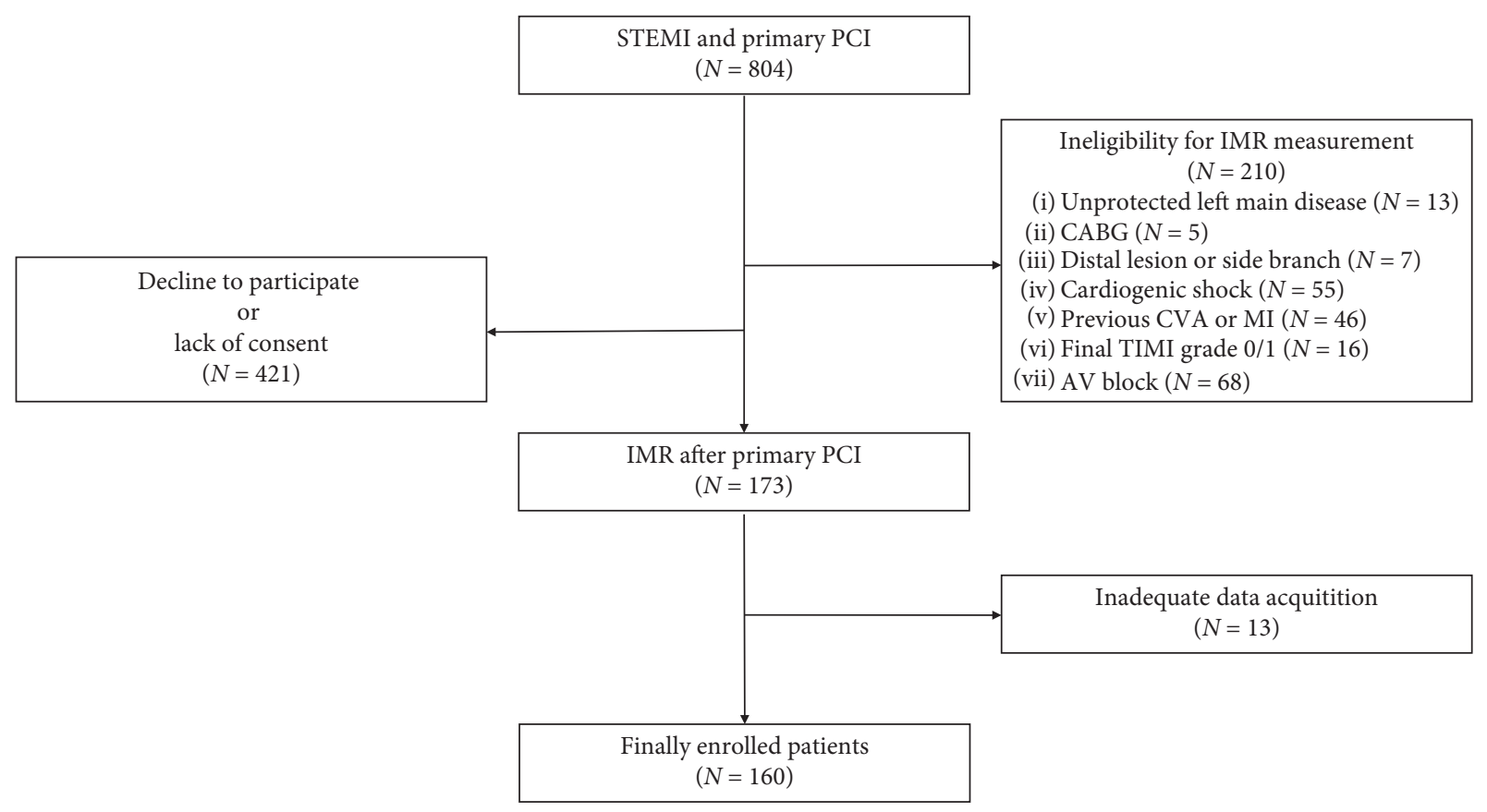

FIgURe 2: Study flowchart.

$P=0.017)$. The use of morphine and GP IIb/IIIa inhibitor was not associated with low IMR value.

In multiple regression analysis for therapeutic strategies using stepwise regression, stent diameter and loading of third-generation P2Y12 inhibitors were negatively correlated with IMR value $(\beta=-9.39, P=0.018$ and $\beta=-10.69$, $P<0.001$, respectively). Postballoon dilatation and the use of GP IIb/IIIa inhibitor were positively correlated with IMR value $(\beta=11.40, \quad P=0.008$ and $\beta=8.51, \quad P=0.013$, respectively).

We analyzed each therapeutic strategy that was significantly associated with IMR value, adjusting for age, sex, and parameters for the previous multiple analysis model in Table 5. Of the stent diameter/length, postballoon dilatation, third-generation P2Y12 inhibitors, and use of GP IIb/IIa inhibitors, only loading of third-generation P2Y12 inhibitors were in significant association with low IMR value $(\beta=-10.28, P<0.013)$. A comparison of IMR according to therapeutic strategies is shown in Figure 3.

\section{Discussion}

The main objective of this study was to determine the therapeutic strategy to reduce MVD in patients with STEMI. The major findings of this study were as follows. (1) Higher Killip class, higher peak troponin-I level, presence of multivessel disease, distal embolization, and delayed reperfusion time were associated with higher IMR value. This finding was consistent with those in previous studies. (2) The mechanical therapeutic strategy had no significant association with lower IMR value. (3) In pharmacological strategies, the preloading of third-generation P2Y12 inhibitors showed a significant association with lower IMR value.
4.1. Clinical Parameters Increasing the Risk of MVD. It has been reported that the burden of ischemic myocardia is a crucial factor in determining the occurrence of MVD [5-9]. The extent of myocardial injury is associated with an increased mortality risk.

Many previous articles show that the IMR provides coronary microcirculation and is independently predictive of LV function and infarct volume and microvascular damage after STEMI [6, 11-14, 23, 24]. Although the exact cut-off value of IMR representing the microvascular damage varies, the key point is that higher IMR value is associated with MVD and ischemic burden of the myocardium. Therefore, high IMR value can represent the MVD of the myocardium.

Previous studies have reported that the occurrence of heart failure (higher Killip class), multivessel disease, and a longer duration from symptom onset was associated with poor prognosis $[15,25]$. Also, the IMR value well correlates with peak troponin-I concentration [24]. Our result is consistent with previous findings, indicating that these clinical factors correlate well with higher IMR values.

TMPG reflected the degree of impaired myocardial perfusion. Impaired TMPG has been associated with a greater coronary thrombus burden, larger infarct size, and poorer salvage indices $[5,26]$. Achieving the final TMPG, grade 3 was associated with lower IMR value in our study, whereas initial and final TIMI grade showed no significant findings on multivariate analysis. Our findings also support that TMPG, which assesses microvascular clearance, was more sensitive to microvascular function than TIMI flow, which reflected epicardial coronary flow. Distal embolization is related to reduced myocardial reperfusion, more extensive myocardial damage, and a poor prognosis [9]. Like previous study results, our data showed that the presence of distal embolization was significantly correlated with higher IMR value. This finding suggests 
TABLE 1: Clinical characteristics of the study population.

\begin{tabular}{|c|c|c|c|c|}
\hline Parameter & Total $(N=160)$ & High IMR $(N=54)$ & Low IMR $(N=106)$ & $P$ value \\
\hline \multicolumn{5}{|l|}{ Clinical characteristics } \\
\hline Age (years) & $56 \pm 11$ & $60 \pm 11$ & $54 \pm 11$ & 0.001 \\
\hline Male, $n(\%)$ & $141(88.1 \%)$ & $45(83.3 \%)$ & $96(90.6 \%)$ & 0.281 \\
\hline Current smoking, $n(\%)$ & $115(71.9 \%)$ & $36(66.7 \%)$ & $79(74.5 \%)$ & 0.353 \\
\hline BMI & $24.6[22.9-26.6]$ & $25.0[21.9-26.9]$ & $24.6[23.4-26.5]$ & 0.734 \\
\hline SBP (mmHg) & $134.9 \pm 23.8$ & $137.7 \pm 25.3$ & $133.5 \pm 23.1$ & 0.299 \\
\hline DBP (mmHg) & $84.4 \pm 16.2$ & $85.3 \pm 17.2$ & $84.0 \pm 15.7$ & 0.637 \\
\hline HR (beats/min) & $76.8 \pm 15.7$ & $73.7 \pm 15.6$ & $78.4 \pm 15.6$ & 0.075 \\
\hline \multicolumn{5}{|l|}{ Killip class } \\
\hline I & $122(76.2 \%)$ & $35(64.8 \%)$ & $87(82.1 \%)$ & \multirow{3}{*}{0.053} \\
\hline II & $32(20.0 \%)$ & $16(29.6 \%)$ & $16(15.1 \%)$ & \\
\hline III & $6(3.8 \%)$ & $3(5.6 \%)$ & $3(2.8 \%)$ & \\
\hline \multicolumn{5}{|l|}{ Medical history, $n(\%)$} \\
\hline Hypertension & $77(48.1 \%)$ & $31(57.4 \%)$ & $46(43.4 \%)$ & 0.131 \\
\hline Diabetes mellitus & $49(30.6 \%)$ & $20(37.0 \%)$ & $29(27.4 \%)$ & 0.283 \\
\hline Dyslipidemia & $68(42.5 \%)$ & $21(38.9 \%)$ & $47(44.3 \%)$ & 0.624 \\
\hline \multicolumn{5}{|l|}{ Time to reperfusion, $\min$} \\
\hline DTB, $\min$ & $68.0[57.0-80.0]$ & $69.5[52.0-83.0]$ & $68.0[59.0-80.0]$ & 0.779 \\
\hline $\mathrm{SHT}, \min$ & $120.0[60.0-210.0]$ & $144.0[71.0-360.0]$ & $95.0[60.0-174.0]$ & 0.003 \\
\hline $\mathrm{SBT}, \min$ & $188.5[125.5-293.0]$ & $235.0[159.0-397.0]$ & $162.0[115.0-265.0]$ & 0.001 \\
\hline \multicolumn{5}{|l|}{ Echocardiographic analysis } \\
\hline LVEDD (mm) & $49.0[46.0-51.0]$ & $48.0[45.0-51.0]$ & $49.0[46.0-51.0]$ & 0.664 \\
\hline LVESD (mm) & $36.0[33.0-38.0]$ & $34.0[32.0-39.0]$ & $36.0[33.0-38.0]$ & 0.489 \\
\hline $\operatorname{LVEF}(\%)$ & $46.5 \pm 7.0$ & $44.7 \pm 6.8$ & $47.3 \pm 7.0$ & 0.028 \\
\hline $\mathrm{E} / \mathrm{e}^{\prime}$ & $10.1[8.4-11.9]$ & $10.5[9.3-12.5]$ & $9.8[8.0-11.2]$ & 0.024 \\
\hline WMSI & $1.6[1.2-1.8]$ & $1.7[1.3-1.8]$ & $1.5[1.2-1.8]$ & 0.040 \\
\hline LV mass (g) & $188.1[158.2-213.6]$ & $193.5[147.8 ; 220.6]$ & $188.1[158.8 ; 207.3]$ & 0.690 \\
\hline LV mass index $\left(\mathrm{g} / \mathrm{m}^{2}\right)$ & $104.8[88.9 ; 116.7]$ & $107.9[89.2 ; 119.3]$ & $103.4[88.9 ; 114.5]$ & 0.424 \\
\hline \multicolumn{5}{|l|}{ Cardiac biomarkers } \\
\hline CK peak, IU/L & $2373.0[774.0-4276.0]$ & $3102.0[1406.0-5580.0]$ & $2020.0[729.0-3897.0]$ & 0.028 \\
\hline CK-MB peak, ng/mL & $224.9[78.2-370.0]$ & $289.4[129.0-465.9]$ & $188.0[73.0-334.0]$ & 0.010 \\
\hline Troponin-I peak, ng/mL & $82.7[21.0-148.4]$ & $100.0[47.0-250.0]$ & $66.0[17.4-122.5]$ & 0.084 \\
\hline NT-proBNP, pg/mL & $70.0[23.0-255.5]$ & $106.0[36.0-293.5]$ & $51.5[21.0-186.0]$ & 0.054 \\
\hline \multicolumn{5}{|l|}{ Renal function } \\
\hline $\mathrm{BUN}, \mathrm{mg} / \mathrm{dL}$ & $15.3[12.9-18.3]$ & $14.9[12.2-18.5]$ & $15.4[13.3-18.1]$ & 0.582 \\
\hline Creatinine, $\mathrm{mg} / \mathrm{dL}$ & $1.0[0.9-1.2]$ & $1.0[0.9-1.2]$ & $1.0[0.9-1.1]$ & 0.543 \\
\hline $\mathrm{eGFR}\left(\mathrm{ml} / \mathrm{min} / 1.73 \mathrm{~m}^{2}\right)$ & $77.5[68.0-91.1]$ & $72.0[62.7-91.2]$ & $79.5[71.3-91.1]$ & 0.081 \\
\hline $\mathrm{eGFR}<60 \mathrm{ml} / \mathrm{min} / 1.73 \mathrm{~m}^{2}, n(\%)$ & $18(11.2 \%)$ & $9(16.7 \%)$ & $9(8.5 \%)$ & 0.184 \\
\hline
\end{tabular}

BMI, body mass index; BUN, blood urea nitrogen; CK, creatine kinase; CK-MB, creatine kinase muscle/brain; DBP, diastolic blood pressure; DTB, door to balloon time; eGFR, estimated glomerular filtration rate; HR, heart rate; IMR, index of microcirculatory resistance; LV, left ventricle; LVEDD, left ventricular end-diastolic diameter; LVEF, left ventricular ejection fraction; LVESD, left ventricular end-systolic diameter; NT-proBNP, N-terminal prohormone of brain natriuretic peptide; SBP, systolic blood pressure; SBT, symptom onset to balloon time; SHT, symptom onset to hospital time; WMSI, wall motion score index.

that an additional therapeutic strategy is necessary to prevent or treat distal embolization during PCI.

4.2. Which Therapeutic Approaches Can Reduce MVD? Reducing periprocedural myocardial injury and enhancing myocardial salvage during primary PCI in patients with STEMI have been a major concern for treatment strategies. Recent studies have shown that distal embolization occurs in $11 \%$ of patients STEMI treated by conventional primary PCI and that occurrence increases the risk of heart failure [27]. Therefore, various mechanical strategies have been developed over the years to reduce distal embolization during primary PCI. However, although most mechanical strategies are still being investigated for potential clinical benefit, their clinical efficacy remains unproven, and their use in primary
PCI routine operations is quite limited [28]. The previous study showed that direct stenting provides better immediate TIMI flow and is a safe and feasible method for selected lesions compared with conventional stenting [29]. However, lesions eligible for direct stenting were shorter and were less complicated than those requiring predilatation. Also, there was a limitation that it was difficult to measure the length of the stent or predict the lesion of stenosis [28]. Direct stenting showed no preventive effect of lowering IMR value in our study. The effect of direct stenting was quite limited and may be eligible only in less complicated culprit lesions.

Various studies have been conducted on the effectiveness of aspiration thrombectomy. However, these promising results did not lead to clinical benefit in the previous randomized trials $[17,30]$. Thus, in the light of current research, the routine use of aspiration thrombectomy is not 
TABLE 2: Coronary angiography findings and physiological assessment of study population.

\begin{tabular}{|c|c|c|c|c|}
\hline Parameter & Total $(N=160)$ & High IMR $(N=54)$ & Low IMR $(N=106)$ & $P$ value \\
\hline $\begin{array}{l}\text { Angiographic parameters } \\
\text { Multivessel, } n(\%)\end{array}$ & $61(38.1 \%)$ & $29(53.7 \%)$ & $32(30.2 \%)$ & 0.006 \\
\hline $\begin{array}{l}\text { Vascular territory, } n(\%) \\
\text { LAD } \\
\text { LCX } \\
\text { RCA }\end{array}$ & $\begin{array}{c}114(71.2 \%) \\
14(8.8 \%) \\
32(20.0 \%) \\
\end{array}$ & $\begin{array}{c}41(75.9 \%) \\
6(11.1 \%) \\
7(13.0 \%) \\
\end{array}$ & $\begin{aligned} & 73(68.9 \%) \\
& 8(7.5 \%) \\
& 25(23.6 \%) \\
&\end{aligned}$ & $\begin{array}{l}0.454 \\
0.647 \\
0.168 \\
\end{array}$ \\
\hline $\begin{array}{l}\text { Initial TIMI grade, } n(\%) \\
\text { TIMI } 0-1 \\
\text { TIMI } 2 \\
\text { TIMI } 3 \\
\end{array}$ & $\begin{array}{l}100(62.5 \%) \\
43(26.9 \%) \\
17(10.6 \%) \\
\end{array}$ & $\begin{array}{c}39(72.2 \%) \\
13(24.1 \%) \\
2(3.7 \%) \\
\end{array}$ & $\begin{array}{l}61(57.5 \%) \\
30(28.3 \%) \\
15(14.2 \%) \\
\end{array}$ & $\begin{array}{l}0.101 \\
0.703 \\
0.079 \\
\end{array}$ \\
\hline $\begin{array}{l}\text { Final TIMI grade, } n(\%) \\
\text { TIMI } 2 \\
\text { TIMI } 3 \\
\end{array}$ & $\begin{array}{c}16(10.0 \%) \\
144(90.0 \%)\end{array}$ & $\begin{array}{l}12(22.2 \%) \\
42(77.8 \%)\end{array}$ & $\begin{array}{c}4(3.8 \%) \\
102(96.2 \%)\end{array}$ & $\begin{array}{l}0.001 \\
0.001 \\
\end{array}$ \\
\hline $\begin{array}{l}\text { Final TMPG, } n(\%) \\
\text { TMPG } 2 \\
\text { TMPG } 3 \\
\end{array}$ & $\begin{array}{c}45(28.1 \%) \\
112(70.0 \%) \\
\end{array}$ & $\begin{array}{l}27(50.0 \%) \\
25(46.3 \%) \\
\end{array}$ & $\begin{array}{l}18(17.0 \%) \\
87(82.1 \%) \\
\end{array}$ & $\begin{array}{l}<0.001 \\
<0.001 \\
\end{array}$ \\
\hline $\begin{array}{l}\text { TIMI thrombus grade, } n \text { (\% } \\
\text { Grade } 1 \\
\text { Grade } 2 \\
\text { Grade } 3 \\
\text { Grade } 4 \\
\text { Grade } 5 \\
\end{array}$ & $\begin{array}{c}4(2.5 \%) \\
12(7.5 \%) \\
25(15.6 \%) \\
36(22.5 \%) \\
83(51.9 \%) \\
\end{array}$ & $\begin{array}{c}2(3.7 \%) \\
3(5.6 \%) \\
4(7.4 \%) \\
13(24.1 \%) \\
32(59.3 \%) \\
\end{array}$ & $\begin{array}{cl}2 & (1.9 \%) \\
9 & (8.5 \%) \\
21 & (19.8 \%) \\
23 & (21.7 \%) \\
51 & (48.1 \%) \\
\end{array}$ & 0.215 \\
\hline $\begin{array}{l}\text { Collateral flow grade, } n(\%) \\
\text { Rentrop } 1 \\
\text { Rentrop } 2 \\
\text { Rentrop } 3 \\
\text { Distal embolization, } n(\%) \\
\text { No reflow, } n(\%)\end{array}$ & $\begin{array}{c}115(71.9 \%) \\
35(21.9 \%) \\
1(0.6 \%) \\
26(16.2 \%) \\
15(9.4 \%) \\
\end{array}$ & $\begin{aligned} & 41(75.9 \%) \\
& 12(22.2 \%) \\
& 0(0.0 \%) \\
& 17(31.5 \%) \\
& 8(14.8 \%) \\
&\end{aligned}$ & $\begin{array}{c}74(69.8 \%) \\
23(21.7 \%) \\
1(0.9 \%) \\
9(8.5 \%) \\
7(6.6 \%) \\
\end{array}$ & $\begin{array}{c}<0.001 \\
0.162 \\
\end{array}$ \\
\hline $\begin{array}{l}\text { Physiologic parameters } \\
\text { Pa (hyp), mm Hg } \\
\text { Pd (hyp), mm Hg } \\
\text { Tmn at rest, sec } \\
\text { Tmn, hyperemia, sec } \\
\text { FFR } \\
\text { CFR } \\
\text { IMR, U } \\
\text { IMRcorr, U }\end{array}$ & $\begin{array}{c}86.0 \pm 16.3 \\
78.5 \pm 15.1 \\
0.5[0.3-0.8] \\
0.3[0.2-0.4] \\
0.9[0.9-1.0] \\
1.6[1.1-2.4] \\
22.1[13.7-34.9] \\
22.4[13.9-35.0]\end{array}$ & $\begin{array}{c}84.9 \pm 17.4 \\
78.7 \pm 15.7 \\
0.7[0.6-1.0] \\
0.6[0.4-0.8] \\
0.9[0.9-1.0] \\
1.3[1.0-1.8] \\
39.8[34.9-52.2] \\
40.2[34.9-52.2]\end{array}$ & $\begin{array}{c}86.5 \pm 15.7 \\
78.5 \pm 14.9 \\
0.4[0.3-0.6] \\
0.2[0.2-0.3] \\
0.9[0.9-1.0] \\
1.8[1.2-2.6] \\
16.5[12.5-22.1] \\
16.4[12.4-22.2]\end{array}$ & $\begin{array}{c}0.564 \\
0.915 \\
<0.001 \\
<0.001 \\
0.256 \\
<0.001 \\
<0.001 \\
<0.001\end{array}$ \\
\hline
\end{tabular}

CFR, coronary flow reserve; FFR, fractional flow reserve; Tmn, mean transit time; IMR, index of microcirculatory resistance; IMRcorr, corrected IMR; LAD, left anterior descending artery; LCX, left circumflex artery; MVD, microvascular dysfunction; Pa (hyp), mean aortic pressure during hyperemia; Pd (hyp), mean distal coronary pressure during hyperemia; RCA, right coronary artery; Tmn, mean transit time; TIMI, thrombolysis in myocardial infarction; TMPG, thrombolysis in myocardial infarction myocardial perfusion grade.

TABLE 3: Clinical outcomes for 3-year MACE in study population.

\begin{tabular}{|c|c|c|c|c|}
\hline Parameter & Total $(N=153)$ & High IMR $(N=52)$ & Low IMR $(N=101)$ & $P$ value \\
\hline 3-years MACE, $n(\%)^{*}$ & $17(11.1 \%)$ & $8(15.4 \%)$ & $9(8.9 \%)$ & 0.279 \\
\hline CV death & $3(2.0 \%)$ & $3(5.8 \%)$ & $0(0.0 \%)$ & 0.038 \\
\hline Hospitalization for $\mathrm{HF}$ & $1(0.7 \%)$ & $1(1.9 \%)$ & $0(0.0 \%)$ & 0.340 \\
\hline TLR & $7(4.6 \%)$ & $1(1.9 \%)$ & $6(5.9 \%)$ & 0.424 \\
\hline Stent thrombosis & $3(2.0 \%)$ & $1(1.9 \%)$ & $2(2.0 \%)$ & 1.000 \\
\hline Nonfatal MI & $0(0.0 \%)$ & $0(0.0 \%)$ & $0(0.0 \%)$ & 1.000 \\
\hline Stroke & $3(2.0 \%)$ & $2(3.8 \%)$ & $1(1.0 \%)$ & 0.267 \\
\hline Major bleeding & $2(1.3 \%)$ & $2(3.8 \%)$ & $0(0.0 \%)$ & 0.114 \\
\hline
\end{tabular}

$\mathrm{CV}$, cardiovascular; HF, heart failure; IMR, index of microcirculatory resistance; MACE, major advanced cardiac events; MI, myocardial infarction; TLR, target lesion revascularization. * MACE was defined as composite of CV death, hospitalization because of HF, TLR, stent thrombosis, nonfatal MI, stroke, and major bleeding. 
TABLE 4: Therapeutic strategies for STEMI in study population.

\begin{tabular}{|c|c|c|c|c|}
\hline Parameter & Total $(N=160)$ & High IMR $(N=54)$ & Low IMR $(N=106)$ & $P$ value \\
\hline \multicolumn{5}{|l|}{ Mechanical strategies } \\
\hline Stent diameter $(\mathrm{mm})$ & $3.0[3.0-3.5]$ & $3.0[2.8-3.5]$ & $3.0[3.0-3.5]$ & 0.248 \\
\hline Stent length $(\mathrm{mm})$ & $24.0[18.0-30.0]$ & $24.0[22.0-33.0]$ & $23.0[18.0-28.0]$ & 0.090 \\
\hline Pre balloon dilatation, $\mathrm{n}(\%)$ & $118(73.8 \%)$ & $37(68.5 \%)$ & $81(76.4 \%)$ & 0.377 \\
\hline Post balloon dilatation, $\mathrm{n}(\%)$ & $20(12.5 \%)$ & $9(16.7 \%)$ & $11(10.4 \%)$ & 0.376 \\
\hline Direct stenting, $n(\%)$ & $42(26.2 \%)$ & $17(31.5 \%)$ & $25(23.6 \%)$ & 0.377 \\
\hline Thrombectomy, $n(\%)$ & $75(46.9 \%)$ & $28(51.9 \%)$ & $47(44.3 \%)$ & 0.464 \\
\hline Thrombectomy + GP IIb/IIIa inhibitor, $n(\%)$ & $31(19.4 \%)$ & $12(22.2 \%)$ & $19(17.9 \%)$ & 0.611 \\
\hline \multicolumn{5}{|l|}{ Pharmacological strategies } \\
\hline Aspirin, $n(\%)$ & $160(100.0 \%)$ & $54(100.0 \%)$ & $106(100.0 \%)$ & 1.000 \\
\hline Clopidogrel, $n(\%)$ & $107(66.9 \%)$ & $43(79.6 \%)$ & $64(60.4 \%)$ & 0.023 \\
\hline Third-generation P2Y12 inhibitors, $n(\%)$ & $53(33.1 \%)$ & $11(20.4 \%)$ & $42(39.6 \%)$ & 0.023 \\
\hline Morphine, $n(\%)$ & $96(60.0 \%)$ & $28(51.9 \%)$ & $68(64.2 \%)$ & 0.183 \\
\hline GP IIb/IIIa inhibitor, $n(\%)$ & $34(21.2 \%)$ & $15(27.8 \%)$ & $19(17.9 \%)$ & 0.216 \\
\hline
\end{tabular}

GP, glycoprotein; IMR, index of microcirculatory resistance.

TABLE 5: Results of multiple linear regression analysis for clinical, echocardiographic, and angiographic factors correlated with IMR value.

\begin{tabular}{|c|c|c|c|c|c|c|}
\hline \multirow{2}{*}{ Parameter } & \multicolumn{3}{|c|}{ Univariable analysis } & \multicolumn{3}{|c|}{ Multiple analysis } \\
\hline & $\beta$ & SE & $P$ value & $\beta$ & SE & $P$ value \\
\hline Age & 0.35 & 0.14 & 0.017 & 0.23 & 0.16 & 0.538 \\
\hline Male & -3.53 & 5.13 & 0.493 & & & \\
\hline Killip class & 13.65 & 3.10 & $<0.001$ & 8.18 & 2.89 & 0.006 \\
\hline SHT $^{*}$ & 5.40 & 1.54 & $<0.001$ & 3.88 & 1.33 & 0.004 \\
\hline LVEF & -0.67 & 0.23 & 0.005 & & & \\
\hline $\mathrm{E} / \mathrm{e}^{\prime}$ & 0.78 & 0.58 & 0.183 & & & \\
\hline WMSI & 17.12 & 5.49 & 0.002 & & & \\
\hline LV mass index & 0.18 & 0.07 & 0.018 & & & \\
\hline CK-MB peak* & 2.64 & 1.55 & 0.093 & & & \\
\hline Troponin-I peak* & 3.91 & 1.44 & 0.008 & 2.52 & 1.22 & 0.042 \\
\hline NT-proBNP* & 5.08 & 1.43 & $<0.001$ & & & \\
\hline Multivessel disease & 11.47 & 3.44 & 0.001 & 9.09 & 2.93 & 0.003 \\
\hline Initial TIMI 0-1 & 5.92 & 3.62 & 0.105 & & & \\
\hline Final TIMI 3 & -5.40 & 6.99 & 0.442 & & & \\
\hline Final TMPG 3 & -8.10 & 3.76 & 0.034 & -5.00 & 3.17 & 0.119 \\
\hline Distal embolization & 14.17 & 4.44 & 0.002 & 8.38 & 3.88 & 0.034 \\
\hline
\end{tabular}

$\beta$, Unstandardized coefficients; CK-MB, creatine kinase muscle/brain; LV, left ventricle; LVEF, left ventricular ejection fraction; NT-proBNP, $N$-terminal prohormone of brain natriuretic peptide; SE, standard error; SHT, symptom onset to hospital time; TIMI, thrombolysis in myocardial infarction; TMPG, thrombolysis in myocardial infarction myocardial perfusion grade; WMSI, wall motion score index. ${ }^{*}$ Data were expressed as quartile $\left(1^{\text {st }} \sim 4^{\text {th }}\right)$.

TABLE 6: Results of univariable and multiple linear regression analysis for therapeutic strategies correlated with IMR value.

\begin{tabular}{|c|c|c|c|c|c|c|c|c|c|}
\hline \multirow{2}{*}{ Parameter } & \multicolumn{3}{|c|}{ Univariable analysis } & \multicolumn{3}{|c|}{ Multiple analysis } & \multicolumn{3}{|c|}{ Multiple analysis* } \\
\hline & $\beta$ & SE & $P$ value & $\beta$ & SE & $P$ value & $\beta$ & SE & $P$ value \\
\hline \multicolumn{10}{|l|}{ Mechanical strategies } \\
\hline Stent diameter & -8.00 & 4.18 & 0.057 & -9.39 & 3.94 & 0.018 & -5.36 & 3.45 & 0.123 \\
\hline Stent length & 0.31 & 0.15 & 0.046 & 0.23 & 0.14 & 0.118 & -0.03 & 0.13 & 0.807 \\
\hline Pre-balloon dilatation & -3.64 & 3.24 & 0.264 & & & & & & \\
\hline Post-balloon dilatation & 10.44 & 4.25 & 0.015 & 11.40 & 4.22 & 0.008 & 8.30 & 3.53 & 0.020 \\
\hline Direct stenting & 3.64 & 3.24 & 0.264 & & & & & & \\
\hline Thrombectomy & 2.60 & 2.87 & 0.366 & & & & & & \\
\hline \multicolumn{10}{|l|}{ Pharmacological strategies } \\
\hline Third-generation P2Y12 inhibitors & -7.24 & 2.99 & 0.017 & -10.69 & 2.91 & $<0.001$ & -10.28 & 2.56 & $<0.001$ \\
\hline Morphine & -4.08 & 2.92 & 0.164 & & & & & & \\
\hline GP IIb/IIIa inhibitor & 8.49 & 3.48 & 0.016 & 8.51 & 3.38 & 0.013 & -0.98 & 3.50 & 0.756 \\
\hline
\end{tabular}

$\beta$, unstandardized coefficients; GP, glycoprotein; SE, standard error. * Multiple regression analysis adjusted for age, sex, Killip class, symptom onset to hospital time, peak troponin-I level, multivessel disease, final thrombolysis in myocardial infarction myocardial perfusion grade, and presence of distal embolization. 


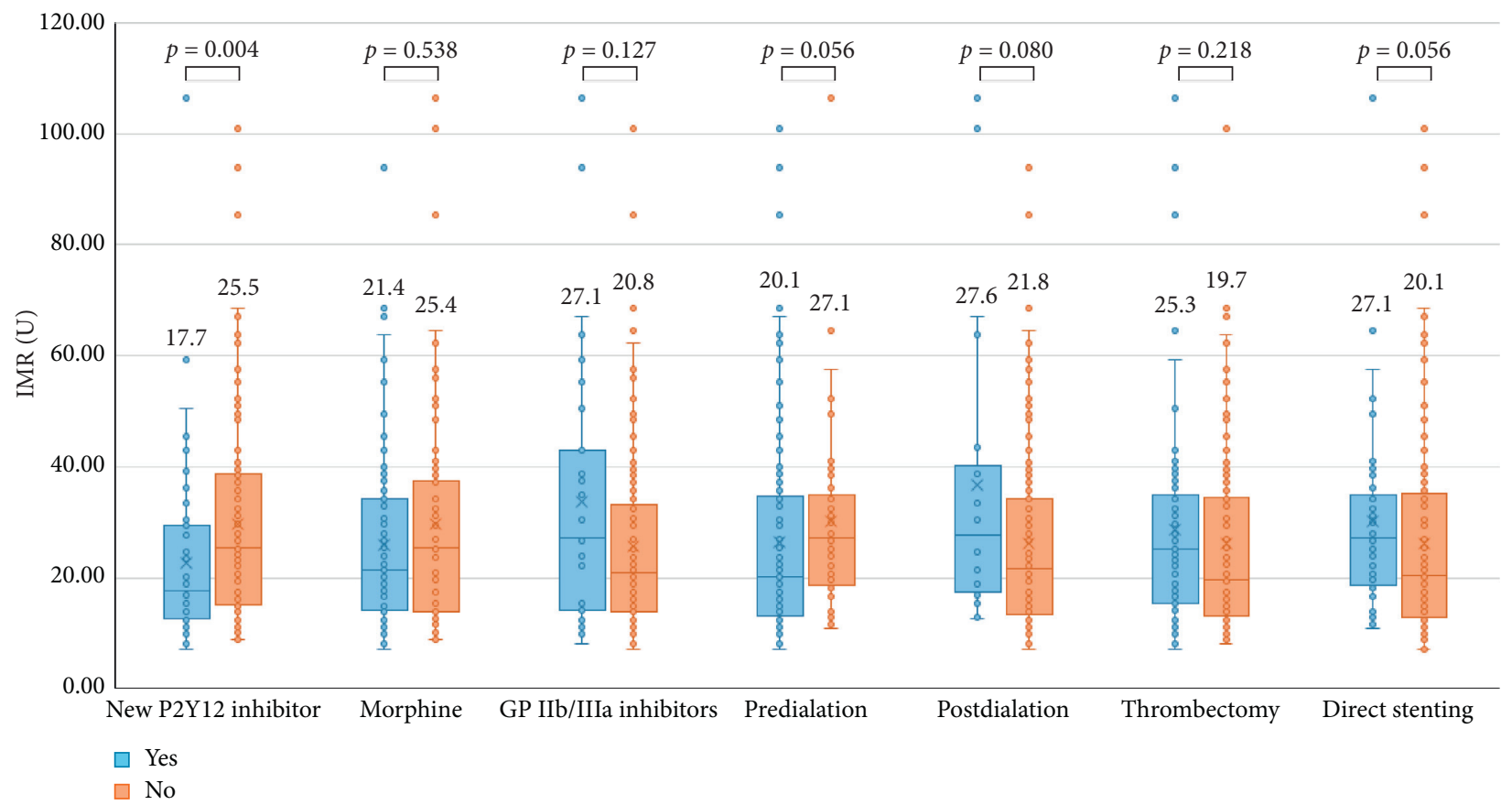

(a)
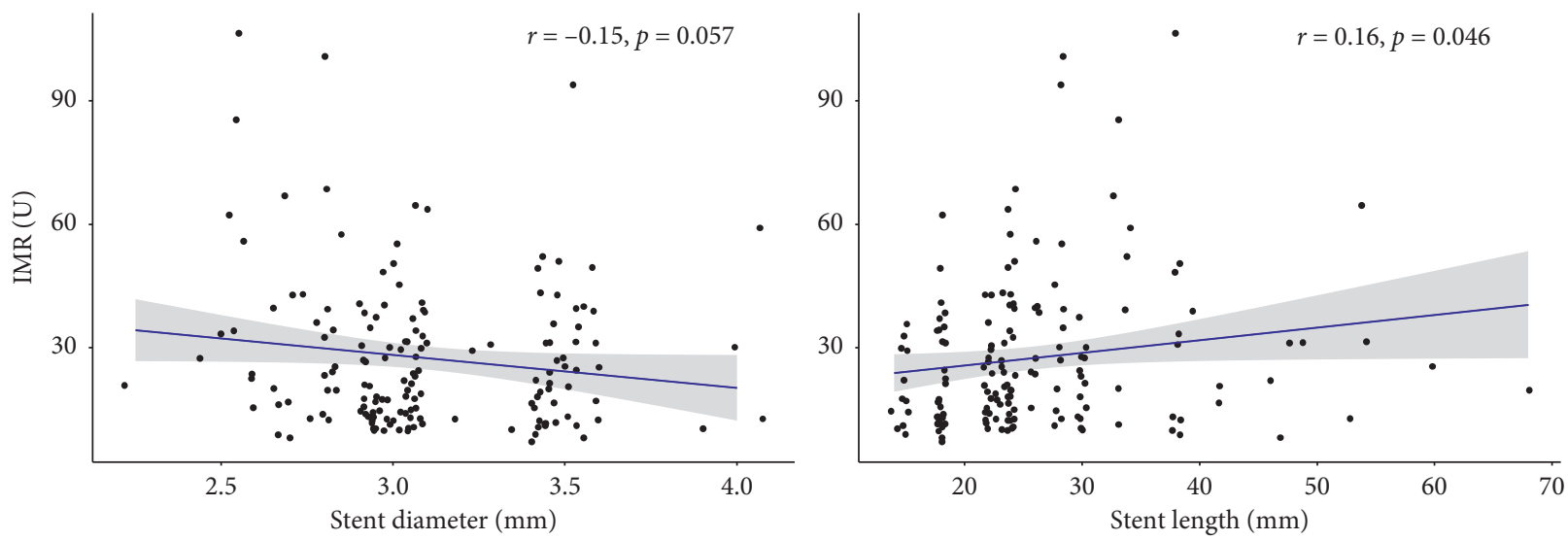

(b)

Figure 3: Comparison of the index of microcirculatory resistance according to therapeutic strategies. (a) Each therapeutic strategy was displayed as box plots. The displayed number means the median value. (b) The association between IMR value and stent diameter/length was displayed as scatter plot.

recommended in recent guidelines [2]. We attempted aspiration thrombectomy alone or combination therapy with GP IIb/IIIa inhibitors. However, these procedural techniques showed no association of lower IMR value.

Although recent meta-analysis showed that intracoronary GP IIb/IIIa inhibitors might be reasonable as bailout therapy in high-risk patients with STEMI, there is no evidence that routine use during primary PCI could reduce myocardial ischemic size [31]. The use of GP IIb/IIIa inhibitors showed no association with IMR value in our study. We speculate that concomitant use with thrombus aspiration or use in patients with no reflow may have reduced the effect of GP IIb/IIIa.

A previous cohort study showed that intravenous morphine infusion had a positive effect on preventing myocardial reperfusion injury. They suggested that morphine inhibits the opening of mitochondrial permeability transition pores (mPTP) mediated by the activation of delta-opioid receptors. Opioid receptor activation-triggered postconditioning may protect the myocardial injury by targeting mPTP [32]. However, another randomized controlled trial demonstrated that morphine did not show the preventive effect of myocardial salvage. They suggested that morphine use is associated with a slower uptake, delayed onset of action, and diminished effects of oral antiplatelet agents [33]. We demonstrated that intravenous morphine use during primary PCI did not differ between the two groups. Our result suggests that morphine did not reduce myocardial infarct burden. However, as there was no clear research on the relationship between morphine use and MVD in patients with STEMI, clarifying their relationship must be investigated by future studies. 
Third-generation P2Y12 inhibitors, prasugrel and ticagrelor, are recommended based on improved clinical outcomes and more potent platelet inhibition compared with clopidogrel in acute coronary syndrome (ACS) [34]. When compared with potent inhibition of platelet function, ticagrelor and prasugrel inhibit approximately 94\% and 90\%, respectively, in those with aspirin, whereas clopidogrel typically achieves a maximum of only $50 \%$ platelet inhibition in combination with aspirin in ACS. Ticagrelor and prasugrel are faster than clopidogrel in reaching its peak concentration (peak effect after loading dose at 2 hours for ticagrelor, 4 hours for prasugrel, and 6 hours for clopidogrel) and has been shown to increase adenosine plasma concentration associated with an inhibition of adenosine uptake of red blood cells, stimulating vasodilatation [35-37]. In our study, microvascular injury was reduced more in the thirdgeneration P2Y12 inhibitors group. Our results support that third-generation P2Y12 inhibitor is more effective in lowering IMR value than clopidogrel in patients with STEMI and this finding represents the protective effect of MVD.

\section{Study Limitation}

Our data did not show any beneficial effect of mechanical strategies on lowering IMR value. Although the larger stent diameter and shorter stent size tend to be associated with lower IMR values in multiple regression analyses which suggested that securing the maximal stent lumen and minimizing the stent length are important for myocardial salvage, our findings suggested that conventional mechanical procedures did not reduce distal embolization effectively. However, as the interventional approach is driven by the operator's decision depending on the nature of the culprit lesion, our mechanical therapeutic strategy cannot be generalized. Thus, selection bias dependent on the interventionist may be reflected in the establishment of treatment strategies, and these variables may play a role as confounders. Moreover, because of data from a relatively small number of patients and a single referral tertiary institute were used, our study participants may be a skewed and selected population based on disease severity and comorbidities, rather than representing the general population. In our study, IMR alone was used to evaluate MVD and no other image tools were used to measure the magnitude of infarct size. Although several studies have shown that IMR is an independent predictor of MVD, radionuclide myocardial perfusion imaging 99mTechnetium Sestamibi single-photon emission tomography and cardiovascular magnetic resonance are still gold standards for measuring myocardial salvage [23]. Further studies would need to be done to evaluate whether the relationship between our therapeutic strategies and MVD hold true in a larger study population, over a long-term follow-up period.

\section{Conclusions}

In our study, mechanical strategies were suboptimal in achieving myocardial salvage. Only preloading of thirdgeneration P2Y12 inhibitors was associated with low IMR value which represents a trend of MVD prevention in STEMI patients. Therefore, it is necessary to use thirdgeneration P2Y12 inhibitors according to the current guidelines, and novel procedural techniques should be developed to reduce MVD in patients with STEMI.

\section{Data Availability}

The data used to support the findings of this study are available from the corresponding author upon request.

\section{Disclosure}

The abstract of the manuscript was presented as Abstract Presentation at the TCTAP 2018, the 23rd Cardiovascular Summit, April 28-May 1, 2018 (Abstract A-125 Journal of the American College of Cardiology. 2018; 71(16):S15).

\section{Conflicts of Interest}

The authors declare that there are no conflicts of interest regarding the publication of this paper.

\section{Acknowledgments}

This work was supported by Inha University Research Grant.

\section{References}

[1] F. Cuculi, A. R. De Caterina, R. K. Kharbanda, and A. P. Banning, "Optimal reperfusion in ST-elevation myocardial infarction-the role of the coronary microcirculation," Swiss Medical Weekly, vol. 141, no. 4950, Article ID w13313, 2011.

[2] B. Ibanez, S. James, S. Agewall et al., "2017 ESC guidelines for the management of acute myocardial infarction in patients presenting with ST-segment elevation: the task force for the management of acute myocardial infarction in patients presenting with ST-segment elevation of the European society of cardiology (ESC)," European Heart Journal, vol. 39, no. 2, pp. 119-177, 2018.

[3] P. T. O'Gara, F. G. Kushner, D. D. Ascheim et al., "2013 ACCF/AHA guideline for the management of ST-elevation myocardial infarction: a report of the American college of cardiology foundation/American heart association task force on practice guidelines," Journal of the American College of Cardiology, vol. 61, no. 4, pp. e78-e140, 2013.

[4] S.-I. Woo, S.-D. Park, D.-H. Kim et al., "Thrombus aspiration during primary percutaneous coronary intervention for preserving the index of microcirculatory resistance: a randomised study," EuroIntervention, vol. 9, no. 9, pp. 1057-1062, 2014.

[5] G. Niccoli, G. Scalone, A. Lerman, and F. Crea, "Coronary microvascular obstruction in acute myocardial infarction," European Heart Journal, vol. 37, no. 13, pp. 1024-1033, 2016.

[6] M. Van Kranenburg, M. Magro, H. Thiele et al., "Prognostic value of microvascular obstruction and infarct size, as measured by CMR in STEMI patients," JACC: Cardiovascular Imaging, vol. 7, no. 9, pp. 930-939, 2014.

[7] G. Niccoli, F. Burzotta, L. Galiuto, and F. Crea, "Myocardial no-reflow in humans," Journal of the American College of Cardiology, vol. 54, no. 4, pp. 281-292, 2009. 
[8] M. Perazzolo Marra, J. A. C. Lima, and S. Iliceto, "MRI in acute myocardial infarction," European Heart Journal, vol. 32, no. 3, pp. 284-293, 2011.

[9] J. Henriques, F. Zijlstra, J. P. Ottervanger et al., "Incidence and clinical significance of distal embolization during primary angioplasty for acute myocardial infarction," European Heart Journal, vol. 23, no. 14, pp. 1112-1117, 2002.

[10] A. S. C. Yong and W. F. Fearon, "Coronary microvascular dysfunction after ST-segment-elevation myocardial infarction," Circulation: Cardiovascular Interventions, vol. 6, no. 3, pp. 201-203, 2013.

[11] W. F. Fearon, M. Shah, M. Ng et al., "Predictive value of the index of microcirculatory resistance in patients with STsegment elevation myocardial infarction," Journal of the American College of Cardiology, vol. 51, no. 5, pp. 560-565, 2008.

[12] W. F. Fearon, L. B. Balsam, H. M. O. Farouque et al., "Novel index for invasively assessing the coronary microcirculation," Circulation, vol. 107, no. 25, pp. 3129-3132, 2003.

[13] M. Faustino, S. B. Baptista, A. Freitas et al., "The index of microcirculatory resistance as a predictor of echocardiographic left ventricular performance recovery in patients with ST-elevation acute myocardial infarction undergoing successful primary angioplasty," Journal of Interventional Cardiology, vol. 29, no. 2, pp. 137-145, 2016.

[14] S. G. Ahn, O. Y. Hung, J.-W. Lee et al., "Combination of the thermodilution-derived index of microcirculatory resistance and coronary flow reserve is highly predictive of microvascular obstruction on cardiac magnetic resonance imaging after ST-segment elevation myocardial infarction," JACC: Cardiovascular Interventions, vol. 9, no. 8, pp. 793-801, 2016.

[15] Y.-S. Baek, S.-D. Park, S.-H. Kim et al., "Clinical and angiographic predictors of microvascular dysfunction in STsegment elevation myocardial infarction," Yonsei Medical Journal, vol. 56, no. 5, pp. 1235-1243, 2015.

[16] S. S. Jolly, S. James, V. Džavík et al., "Thrombus aspiration in ST-segment-elevation myocardial infarction," Circulation, vol. 135, no. 2, pp. 143-152, 2017.

[17] O. Fröbert, B. Lagerqvist, G. K. Olivecrona et al., "Thrombus aspiration during ST-segment elevation myocardial infarction," New England Journal of Medicine, vol. 369, no. 17, pp. 1587-1597, 2013.

[18] L. Iannetta, P. E. Puddu, D. Cuturello, A. Saladini, M. Pellicano, and M. Schiariti, "Is there still a role for glycoprotein IIb/IIIa antagonists in acute coronary syndromes?” Cardiology Research, vol. 4, no. 1, pp. 1-7, 2013.

[19] R. M. Lang, L. P. Badano, V. Mor-Avi et al., "Recommendations for cardiac chamber quantification by echocardiography in adults: an update from the American society of echocardiography and the European association of cardiovascular imaging," Journal of the American Society of Echocardiography, vol. 28, no. 1, pp. 1-39, 2015.

[20] G. Sianos, M. I. Papafaklis, J. Daemen et al., "Angiographic stent thrombosis after routine use of drug-eluting stents in ST-segment elevation myocardial infarction," Journal of the American College of Cardiology, vol. 50, no. 7, pp. 573-583, 2007.

[21] K. P. Rentrop, M. Cohen, H. Blanke, and R. A. Phillips, "Changes in collateral channel filling immediately after controlled coronary artery occlusion by an angioplasty balloon in human subjects," Journal of the American College of Cardiology, vol. 5, no. 3, pp. 587-592, 1985.

[22] N. H. J. Pijls, B. De Bruyne, L. Smith et al., "Coronary thermodilution to assess flow reserve," Circulation, vol. 105, no. 21, pp. 2482-2486, 2002.
[23] H. E. Botker, A. K. Kaltoft, S. F. Pedersen, and W. Y. Kim, "Measuring myocardial salvage," Cardiovascular Research, vol. 94, no. 2, pp. 266-275, 2012.

[24] R. McGeoch, S. Watkins, C. Berry et al., "The index of microcirculatory resistance measured acutely predicts the extent and severity of myocardial infarction in patients with STsegment elevation myocardial infarction," JACC: Cardiovascular Interventions, vol. 3, no. 7, pp. 715-722, 2010.

[25] L. Vicent, J. Velásquez-Rodríguez, M. J. Valero-Masa et al., "Predictors of high Killip class after ST segment elevation myocardial infarction in the era of primary reperfusion," International Journal of Cardiology, vol. 248, pp. 46-50, 2017.

[26] E. Appelbaum, A. J. Kirtane, A. Clark et al., "Association of TIMI myocardial perfusion grade and ST-segment resolution with cardiovascular magnetic resonance measures of microvascular obstruction and infarct size following ST-segment elevation myocardial infarction," Journal of Thrombosis and Thrombolysis, vol. 27, no. 2, pp. 123-129, 2009.

[27] J. Lønborg, H. Kelbæk, S. Helqvist et al., "The impact of distal embolization and distal protection on long-term outcome in patients with ST elevation myocardial infarction randomized to primary percutaneous coronary intervention-results from a randomized study," European Heart Journal. Acute Cardiovascular Care, vol. 4, no. 2, pp. 180-188, 2015.

[28] G. Ndrepepa and A. Kastrati, "Mechanical strategies to enhance myocardial salvage during primary percutaneous coronary intervention in patients with STEMI," EuroIntervention, vol. 12, no. 3, pp. 319-328, 2016.

[29] M. Möckel, J. Vollert, A. J. Lansky et al., "Comparison of direct stenting with conventional stent implantation in acute myocardial infarction," The American Journal of Cardiology, vol. 108, no. 12, pp. 1697-1703, 2011.

[30] S. S. Jolly, J. A. Cairns, S. Yusuf et al., "Randomized trial of primary PCI with or without routine manual thrombectomy," New England Journal of Medicine, vol. 372, no. 15, pp. 1389-1398, 2015.

[31] S. Friedland, M. J. Eisenberg, and A. Shimony, "Meta-analysis of randomized controlled trials of intracoronary versus intravenous administration of glycoprotein IIb/IIIa inhibitors during percutaneous coronary intervention for acute coronary syndrome," The American Journal of Cardiology, vol. 108, no. 9, pp. 1244-1251, 2011.

[32] I. Rentoukas, G. Giannopoulos, A. Kaoukis et al., "Cardioprotective role of remote ischemic periconditioning in primary percutaneous coronary intervention," JACC: Cardiovascular Interventions, vol. 3, no. 1, pp. 49-55, 2010.

[33] H. B. Gwag, E. K. Kim, T. K. Park et al., "Cardioprotective effects of intracoronary morphine in ST-segment elevation myocardial infarction patients undergoing primary percutaneous coronary intervention: a prospective, randomized trial," Journal of the American Heart Association, vol. 6, no. 4, Article ID e005426, 2017.

[34] L. Wallentin, R. C. Becker, A. Budaj et al., "Ticagrelor versus clopidogrel in patients with acute coronary syndromes," New England Journal of Medicine, vol. 361, no. 11, pp. 1045-1057, 2009.

[35] J. N. Khan, J. P. Greenwood, S. A. Nazir et al., "Infarct size following treatment with second-versus third-generation P2Y12 antagonists in patients with multivessel coronary disease at ST-segment elevation myocardial infarction in the cv LPRIT study," Journal of the American Heart Association, vol. 5, no. 6, Article ID e003403, 2016.

[36] J. L. Winter, D. S. Lindefjeld, N. Veas et al., "Angiographic and electrocardiographic parameters of myocardial reperfusion in angioplasty of patients with ST elevation acute myocardial 
infarction loaded with ticagrelor or clopidogrel (MICAMITICLO trial)," Cardiovascular Revascularization Medicine, vol. 15, no. 5, pp. 284-288, 2014.

[37] E. Cerrato, A. Quiros, M. Echavarria-Pinto et al., "Protective effect on the coronary microcirculation of patients with diabetes by clopidogrel or ticagrelor (PREDICT): study rationale and design. a randomized multicenter clinical trial using intracoronary multimodal physiology," Cardiovascular Diabetology, vol. 16, no. 1, p. 68, 2017. 